Article

\title{
A note on Jeśmanowicz' conjecture for non-primitive Pythagorean triples
}

\author{
Van Thien Nguyen ${ }^{1}$, Viet Kh. Nguyen ${ }^{2}$ and Pham Hung Quy ${ }^{1, *}$ \\ 1 Department of Mathematics, Hoa Lac High Tech Park, FPT University, Hanoi, Vietnam. \\ 2 Department of Mathematics and Information Assurance, Hoa Lac High Tech Park, FPT University, Hanoi, Vietnam. \\ * Correspondence: quyph@fe.edu.vn \\ Communicated by: Mujahid Abbas \\ Received: 6 January 2021; Accepted: 2 March 2021; Published: 21 March 2021.
}

\begin{abstract}
Let $(a, b, c)$ be a primitive Pythagorean triple parameterized as $a=u^{2}-v^{2}, b=2 u v, c=u^{2}+v^{2}$, where $u>v>0$ are co-prime and not of the same parity. In 1956, L. Jeśmanowicz conjectured that for any positive integer $n$, the Diophantine equation $(a n)^{x}+(b n)^{y}=(c n)^{z}$ has only the positive integer solution $(x, y, z)=(2,2,2)$. In this connection we call a positive integer solution $(x, y, z) \neq(2,2,2)$ with $n>1$ exceptional. In 1999 M.-H. Le gave necessary conditions for the existence of exceptional solutions which were refined recently by H. Yang and R.-Q. Fu. In this paper we give a unified simple proof of the theorem of Le-Yang-Fu. Next we give necessary conditions for the existence of exceptional solutions in the case $v=2, u$ is an odd prime. As an application we show the truth of the Jeśmanowicz conjecture for all prime values $u<100$.
\end{abstract}

Keywords: Diophantine equations; Non-primitive Pythagorean triples; Jeśmanowicz conjecture.

MSC: 11D61; 11D41.

\section{Introduction}

$\mathbf{L}$

et $(a, b, c)$ be a primitive Pythagorean triple. Clearly for such a triple with $2 \mid b$ one has the following parameterization

$$
a=u^{2}-v^{2}, b=2 u v, c=u^{2}+v^{2}
$$

with

$$
u>v>0, \operatorname{gcd}(u, v)=1, u+v \equiv 1(\bmod 2) .
$$

In 1956 L. Jeśmanowicz ([1]) made the following conjecture:

Conjecture 1. For any positive integer $n$, the Diophantine equation

$$
(a n)^{x}+(b n)^{y}=(c n)^{z}
$$

has only the positive integer solution $(x, y, z)=(2,2,2)$.

The primitive case of the conjecture $(n=1)$ was investigated thoroughly. Although the conjecture is still open, many special cases are shown to be true. We refer to a recent survey [2] for a detailed account.

Much less known about the non-primitive case $(n>1)$. A positive integer solution $(x, y, z, n)$ of $(2)$ is called exceptional if $(x, y, z) \neq(2,2,2)$ and $n>1$. For a positive integer $t$, let $\mathcal{P}(t)$ denote the set of distinct prime factors of $t$ and $P(t)$ - their product. The first known result in this direction was obtained in 1998 by M.-J. Deng and G.L. Cohen ([3]), namely if $u=v+1, a$ is a prime power, and either $P(b) \mid n$, or $P(n) \nmid b$, then (2) has only positive integer solution $(x, y, z)=(2,2,2)$. In 1999, M.-H. Le gave necessary conditions for (2) to have exceptional solutions.

Theorem 1. [4] If $(x, y, z, n)$ is an exceptional solution of (2), then one of the following three conditions is satisfied:

(i) $\max \{x, y\}>\min \{x, y\}>z, \mathcal{P}(n) \varsubsetneqq \mathcal{P}(c)$; 
(ii) $x>z>y, \mathcal{P}(n) \subset \mathcal{P}(b)$;

(iii) $y>z>x, \mathcal{P}(n) \subset \mathcal{P}(a)$.

However, as noted in [5] by H. Yang and R.-Q. Fu, the case $x=y>z$ is not completely handled by the arguments used in [4]. Furthermore they completed the unhandled case ([5], Theorem 1) based on a powerful result of Zsigmondy ([6], cf. [7,8]). In fact one can give a unified simple proof of Theorem of Le-Yang-Fu (Theorem 1) by using a weaker version of the Zsigmondy theorem as stated in Lemma 3 of [3].

Since many works $[3,4]$ intensively investigated the first interesting family of primitive triples:

$$
v=1, u=2^{k}, k=1,2, \ldots
$$

Most recently, X.-W. Zhang and W.-P. Zhang [9], and T. Miyazaki [10] independently proved Conjecture 1 for the (infinite) family (3).

It is natural to treat the next interesting case: $v=2, u$ is an odd prime which was known recently for few values $u: u=3$ ([3]), $u=5$ - by Z. Cheng, C.-F. Sun and X.-N. Du, $u=7-$ by C.-F. Sun, Z. Cheng, and by G. Tang, $u=11$ - by W.-Y. Lu, L. Gao and H.-F. Hao (cf. [2] for references). Let's formulate our main results. We rewrite (2) as

$$
\left[\left(u^{2}-4\right) n\right]^{x}+(4 u n)^{y}=\left[\left(u^{2}+4\right) n\right]^{z} .
$$

An arithmetical argument (given in Lemma 7 below) shows that $u^{2}-4$ admits a proper decomposition $u^{2}-4=u_{1} u_{2}, \operatorname{gcd}\left(u_{1}, u_{2}\right)=1$, so that there are three possibilities to consider: $u_{1} \equiv \pm 1,5(\bmod 8)$.

Theorem 2. If $(x, y, z, n)$ is an exceptional solution of $(4)$ and $u_{1} \equiv \pm 1(\bmod 8)$, then $y$ is even.

In view of Theorem 2 the possibility $u_{1} \equiv-1(\bmod 8)$ is eliminated, because in this case $x, y, z$ are even, which is in general impossible by an auxiliary argument (Lemma 8 below).

Let $v_{q}(t)$, for a prime $q$, denote the exponent of $q$ in the prime factorization of $t$, and let $\left(\frac{m}{m}\right)$ denote the Jacobi quadratic residue symbol.

Theorem 3. If $(x, y, z, n)$ is an exceptional solution of (4), then one of the following cases is satisfied

(1) $v_{2}\left(u_{1}-1\right)=3:\left(v_{2}(x), v_{2}(y), v_{2}(z)\right)=(0, \geq 2,1) ; u_{1}$ admits a proper decomposition $u_{1}=t_{1} t_{2}, \operatorname{gcd}\left(t_{1}, t_{2}\right)=1$ and $t_{1}, t_{2} \equiv 5(\bmod 8)$ satisfying certain special Diophantine equations;

(2) $u_{1} \equiv 5(\bmod 8), u_{2}=w^{2^{s}}$, where $s=v_{2}(z-x)-v_{2}(x)$ and either of the following

(2.1) $w \equiv \pm 3(\bmod 8):\left(v_{2}(x), v_{2}(y), v_{2}(z)\right)=(0, \geq 1,0) ; u \equiv 1(\bmod 4) ;\left(\frac{u_{1}}{p}\right)=\left(\frac{w}{p}\right), \forall p \mid\left(u^{2}+4\right)$ and $\left(\frac{w}{p}\right)=\left(\frac{u^{2}+4}{p}\right), \forall p \mid u_{1}$

(2.2) $w \equiv \pm 1(\bmod 8):\left(v_{2}(x), v_{2}(y), v_{2}(z)\right)=(\beta, 0, \beta), \beta \geq 1 ; u \equiv \pm 3(\bmod 8) ;\left(\frac{w}{p}\right)=1, \forall p \mid\left(u^{2}+4\right)$ and $\left(\frac{w}{p}\right)=\left(\frac{u}{p}\right), \forall p \mid u_{1}$. Moreover, if $u \equiv 3(\bmod 8)$, then $w$ can not be a square.

Corollary 1. Conjecture 1 is true for $v=2, u$-an odd prime $<100$.

Let's explain the ideas in proving our main results. As for Theorem 2 and Theorem 3 we exploit a total analysis of Jacobi quadratic and quartic residues. In the case $u_{1} \equiv 1(\bmod 8)$ we have a further proper decomposition $u_{1}=t_{1} t_{2}$, which leads to certain special Diophantine equations. Theorem 3 helps us substantially in reducing the verification process, as the possibility $u_{1} \equiv 5(\bmod 8)$ occurs quite sparsely. We demonstrate this for $u<100$ in proving Corollary 1.

The paper is organized as follows. In Section 2 we give a unified simple proof of Theorem 1. Section 3 provides some reduction of the problem and preliminary results. Theorem 2 will be proved in Section 4 . The case $u_{1} \equiv 5(\bmod 8)$ and Theorem 3 will be treated in Section 5 . The verification for $u<100$ in Corollary 1 will be given in the last Section 6 .

\section{A Simple Proof of Theorem 1}

We shall use the following weaker version of Zsigmondy's theorem. 
Lemma 1. (cf. [3], Lemma 3) For $X>Y>0$ co-prime integers,

(1) if $q$ is a prime, then

$$
\operatorname{gcd}\left(X-Y, \frac{X^{q}-Y^{q}}{X-Y}\right)=1, \text { or } q
$$

(2) if $q$ is an odd prime, then

$$
\operatorname{gcd}\left(X+Y, \frac{X^{q}+Y^{q}}{X+Y}\right)=1, \text { or } q
$$

Proof. Part (2) is Lemma 3 of [3]. As for part (1) one argues similarly: if $\ell^{r}$ is a common prime power divisor of $X-Y$ and $\left(X^{q}-Y^{q}\right) /(X-Y)$. Clearly

$$
\frac{X^{q}-Y^{q}}{X-Y} \equiv 0\left(\bmod \ell^{r}\right)
$$

On the other hand from the fact that $X \equiv Y\left(\bmod \ell^{r}\right)$ it follows

$$
\frac{X^{q}-Y^{q}}{X-Y}=X^{q-1}+X^{q-2} Y+\cdots+X Y^{q-2}+Y^{q-1} \equiv q Y^{q-1}\left(\bmod \ell^{r}\right) .
$$

Since $\ell \nmid Y$, (5)-(6) imply that $\ell=q$, and $r=1$.

Remark 1. Part (1) of Lemma 1 is a special case of Theorem IV in [7].

Lemma 2. For a prime divisor $q$ of $(X-Y)$ and positive integer $\beta$

$$
v_{q}\left(X^{q^{\beta}}-Y^{q^{\beta}}\right)=\beta+v_{q}(X-Y) .
$$

Proof. Applying part (1) of Lemma $1 \beta$ times one has

$$
\begin{gathered}
\operatorname{gcd}\left(X^{q^{\beta-1}}-Y^{q^{\beta-1}}, \frac{X^{q^{\beta}}-Y^{q^{\beta}}}{X^{q^{\beta-1}}-Y^{q^{\beta-1}}}\right)=q ; \\
\cdots \\
\operatorname{gcd}\left(X-Y, \frac{X^{q}-Y^{q}}{X-Y}\right)=q .
\end{gathered}
$$

Hence the formula (7).

In view of Lemma 2 of [3] there are no exceptional solutions with $z \geq \max \{x, y\}$, so as in [4] we have to eliminate the following three cases:

$$
\begin{aligned}
& \text { (I) } x>y=z \\
& \text { (II) } y>x=z \\
& \text { (III) } x=y>z \\
& \text { (I) } x>y=z \text { : Dividing both sides of (2) by } n^{y} \text { one gets }
\end{aligned}
$$

$$
a^{x} n^{x-y}=c^{y}-b^{y} .
$$

By considering mod $c+b$, and taking into account $(c+b)(c-b)=a^{2}$, one sees that $y$ must be even, say $y=2 y_{1}$. Now put $X=c^{2}, Y=b^{2}$, so $X \equiv Y\left(\bmod a^{2}\right), \operatorname{gcd}(Y, a)=1$. Taking $\bmod a$ and in view of (8)

$$
0 \equiv \frac{X^{y_{1}}-Y^{y_{1}}}{X-Y}=X^{y_{1}-1}+X^{y_{1}-2} Y+\cdots+X Y^{y_{1}-2}+Y^{y_{1}-1} \equiv y_{1} Y^{y_{1}-1}(\bmod a)
$$

one concludes that $a \mid y_{1}$.

For any $q \in \mathcal{P}(a)$ let $\beta=v_{q}\left(y_{1}\right)$, so that $y_{1}=q^{\beta} y_{2}$ with $q \nmid y_{2}$. Putting $U=X^{q^{\beta}}, V=Y^{q^{\beta}}$ for short, we have

$$
X^{y_{1}}-Y^{y_{1}}=(U-V)\left(U^{y_{2}-1}+U^{y_{2}-2} V+\cdots+U V^{y_{2}-2}+V^{y_{2}-1}\right),
$$


and

$$
U^{y_{2}-1}+U^{y_{2}-2} V+\cdots+U V^{y_{2}-2}+V^{y_{2}-1} \equiv y_{2} V^{y_{2}-1} \not \equiv 0(\bmod q) .
$$

Lemma 2 and (9), (10) imply that

$$
v_{q}\left(X^{y_{1}}-Y^{y_{1}}\right)=v_{q}(U-V)=\beta+2 v_{q}(a) .
$$

In view of (8) the equality (11) means that $a^{x-2} \mid y_{1}$ in contradiction with $y_{1}=y / 2<a^{x-2}$ as $x>y, a>1$. (II) $y>x=z$ : Similarly dividing both sides of (2) by $n^{z}$ one gets

$$
b^{y} n^{y-x}=c^{x}-a^{x} .
$$

Arguing as above with mod $c+a$, one sees that $x$ must be even, say $x=2 x_{1}$. Put $X=c^{2}, Y=a^{2}$. Considering mod $b$ and from (12) it follows that $b \mid x_{1}$. So $v_{q}\left(X^{x_{1}}-Y^{x_{1}}\right)=v_{q}\left(x_{1}\right)+2 v_{q}(b)$ for any $q \in \mathcal{P}(b)$, therefore $b^{y-2} \mid x_{1}$ in contradiction with $x_{1}=x / 2<b^{y-2}$ as $y>x, b>1$.

(III) $x=y>z$ : Dividing both sides of (2) by $n^{z}$ one gets

$$
\left(a^{x}+b^{x}\right) n^{x-z}=c^{z}
$$

First we claim that $x$ must be even. Indeed, if $x$ is odd, then from (13) it follows that there is an odd prime $q \in \mathcal{P}(a+b) \cap \mathcal{P}(c)$, so $q \in \mathcal{P}(a b)$, as $c^{2}=a^{2}+b^{2}$. A contradiction with $\operatorname{gcd}(a, b)=1$.

Writing now $x=2 x_{1}$ one sees that $x_{1}$ must be odd. Since otherwise for an odd prime $q \in \mathcal{P}\left(a^{x}+b^{x}\right) \cap \mathcal{P}(c)$ taking $\bmod q$ and by (13)

$$
0 \equiv a^{x}+b^{x}=a^{2 x_{1}}+\left(c^{2}-a^{2}\right)^{x_{1}} \equiv 2 a^{2 x_{1}}(\bmod q),
$$

one gets a contradiction with $\operatorname{gcd}(a, c)=1$.

Now from (13) we see that

$$
\frac{\left(a^{2}\right)^{x_{1}}+\left(b^{2}\right)^{x_{1}}}{a^{2}+b^{2}}=\frac{c^{z-2}}{n^{x-z}}>1 .
$$

as $x>z \geq 2$. So there is an odd prime $q \in \mathcal{P}(c)$ dividing $\left(\left(a^{2}\right)^{x_{1}}+\left(b^{2}\right)^{x_{1}}\right) /\left(a^{2}+b^{2}\right)$. Considering mod $q$ and taking into account $a^{2} \equiv-b^{2} \bmod q, q \nmid a$ one has

$$
0 \equiv \frac{\left(a^{2}\right)^{x_{1}}+\left(b^{2}\right)^{x_{1}}}{a^{2}+b^{2}}=\left(a^{2}\right)^{x_{1}-1}-\left(a^{2}\right)^{x_{1}-2} b^{2}+\cdots-a^{2}\left(b^{2}\right)^{x_{1}-2}+\left(b^{2}\right)^{x_{1}-1} \equiv x_{1} a^{2 x_{1}-2}(\bmod q) .
$$

Hence $q \mid x_{1}$, and so $\left(\left(a^{2}\right)^{q}+\left(b^{2}\right)^{q}\right) \mid\left(\left(a^{2}\right)^{x_{1}}+\left(b^{2}\right)^{x_{1}}\right)$. Applying part (1) of Lemma 1 we get

$$
\operatorname{gcd}\left(a^{2}+b^{2}, \frac{\left(a^{2}\right)^{q}+\left(b^{2}\right)^{q}}{a^{2}+b^{2}}\right)=q .
$$

On the other hand from (14) one knows that $\left(\left(a^{2}\right)^{q}+\left(b^{2}\right)^{q}\right) /\left(a^{2}+b^{2}\right)$ is a product of primes in $\mathcal{P}(c)$. It is easy to see that $\left(\left(a^{2}\right)^{q}+\left(b^{2}\right)^{q}\right) /\left(a^{2}+b^{2}\right)>q$. So either $v_{q}\left(\left(\left(a^{2}\right)^{q}+\left(b^{2}\right)^{q}\right) /\left(a^{2}+b^{2}\right)\right) \geq 2$ and $v_{q}\left(a^{2}+b^{2}\right) \geq 2$, or both of them must have another common prime factor in $\mathcal{P}(c)$, a contradiction with (15).

\section{Preliminary reduction}

We need some reduction of the problem. The following result is due to N. Terai [11].

Lemma 3. Conjecture 1 is true for $n=1, v=2$.

Because of Lemma 3 we will assume henceforth $n>1$.

M.-J. Deng ([12], from the proof of Lemma 2), and H. Yang, R.-Q. Fu ([5]) showed that we can remove the condition $(i)$ in Theorem 1.

Lemma 4. If $(x, y, z, n)$ is an exceptional solution, then either $x>z>y$, or $y>z>x$. 
Note that the proof of Lemma 4 relies essentially on the condition $n>1$. It could be interesting to find a proof of this result for the case $n=1$.

Furthermore, in the case when $u$ is an odd prime and $v=2$, H. Yang, R.-Q. Fu [13] succeeded to eliminate the possibility (ii) in Theorem 1.

Lemma 5. Suppose that $u$ is an odd prime and $v=2$. Then equation (2) has no exceptional solutions $(x, y, z, n)$ with $x>z>y$.

Lemma 6. For a positive integer $w$

(1) if $v_{2}(w) \geq 2$, then $v_{2}\left[(1+w)^{x}-1\right]=v_{2}(w)+v_{2}(x)$;

(2) if $v_{2}(w)=1$ and $x$ is odd, then $v_{2}\left[(1+w)^{x}-1\right]=1$;

(3) if $v_{2}(w)=1$ and $x$ is even, then $v_{2}\left[(1+w)^{x}-1\right]=v_{2}(2+w)+v_{2}(x)$.

In particular $v_{2}\left[(1+w)^{x}-1\right]=2+v_{2}(x)$, if $w \equiv 4(\bmod 8)$; or if $w \equiv 2(\bmod 8)$ and $x$ is even.

Proof. (1) The conclusions of Lemma 6 are true trivially for $x=1$. Assuming now $x \geq 2$ we have

$$
(1+w)^{x}-1=w\left(C_{x}^{1}+C_{x}^{2} w+\cdots+C_{x}^{x-1} w^{x-2}+C_{x}^{x} w^{x-1}\right) .
$$

Clearly $v_{2}(j) \leq j-1$ for $j=2, \cdots, x$, and so

$$
v_{2}\left(C_{x}^{j} w^{j-1}\right)=v_{2}\left(\frac{x}{j} C_{x-1}^{j-1} w^{j-1}\right) \geq v_{2}(x)+j-1>v_{2}(x),
$$

as $v_{2}(w) \geq 2$. Hence the conclusion follows from taking $v_{2}(\cdot)$ on both sides of (16).

(2) Obvious from (16), since $C_{x}^{1}+C_{x}^{2} w+\cdots+C_{x}^{x-1} w^{x-2}+C_{x}^{x} w^{x-1}$ is odd in this case.

(3) Writing $x=2 x_{1}$ we have

$$
(1+w)^{x}-1=\left[(1+w)^{x_{1}}-1\right]\left[(1+w)^{x_{1}}+1\right] .
$$

If $x_{1}$ is odd, i.e., $v_{2}(x)=1$, then $v_{2}\left[(1+w)^{x_{1}}-1\right]=1$ by the part $(2)$ above, and $v_{2}\left[(1+w)^{x_{1}}+1\right]=$ $v_{2}(2+w)$, as

$$
(1+w)^{x_{1}}+1=(2+w)\left[(1+w)^{x_{1}-1}-(1+w)^{x_{1}-2}+\cdots-(1+w)+1\right]
$$

and $(1+w)^{x_{1}-1}-(1+w)^{x_{1}-2}+\cdots-(1+w)+1$ is odd.

If $x_{1}$ is even, then $v_{2}\left[(1+w)^{x_{1}}+1\right]=1$, since

$$
(1+w)^{x_{1}}+1=2+C_{x_{1}}^{1} w+C_{x_{1}}^{2} w^{2}+\cdots+C_{x_{1}}^{x_{1}-1} w^{x_{1}-1}+w^{x_{1}} .
$$

Therefore $v_{2}\left[(1+w)^{x}-1\right]=v_{2}\left[(1+w)^{x_{1}}-1\right]+1$ by $(17)$. Now the descending argument yields the conclusion.

The following claims play a central role in the next sections.

Lemma 7. If $(x, y, z, n)$ is an exceptional solution of (4), then $u^{2}-4$ admits a proper decomposition $u^{2}-4=$ $u_{1} u_{2}, \operatorname{gcd}\left(u_{1}, u_{2}\right)=1$ and with one of the following conditions satisfied:

(1) $u_{1} \equiv 1(\bmod 8)$ and $v_{2}(z)=v_{2}\left(u_{1}-1\right)+v_{2}(x)-2$;

(2) $u_{1} \equiv 7(\bmod 8), v_{2}(z)=v_{2}\left(u_{1}+1\right)+v_{2}(x)-2$, and $v_{2}(x) \geq 1$;

(3) $u_{1} \equiv 5(\bmod 8), u_{2}$ is a square and and $v_{2}(z)=v_{2}(x)$.

Proof. In view of Lemmas 4, 5 we may assume the existence of an exceptional solution with $y>z>x$ (the case (iii) of Theorem 1). Dividing both sides of (4) by $n^{x}$ one gets

$$
\left(u^{2}-4\right)^{x}=\left[\left(u^{2}+4\right)^{z}-(4 u)^{y} n^{y-z}\right] n^{z-x} .
$$


It is easy to see that $\operatorname{gcd}\left(u^{2}+4, n\right)=1$. So (18) is equivalent to the following system

$$
\left\{\begin{array}{l}
\left(u^{2}+4\right)^{z}-(4 u)^{y} n^{y-z}=u_{1}^{x} \\
n^{z-x}=u_{2}^{x}
\end{array}\right.
$$

with $u^{2}-4=u_{1} u_{2}, \operatorname{gcd}\left(u_{1}, u_{2}\right)=1$. The system (19) can be rewritten as

$$
\left(u^{2}+4\right)^{z}-2^{2 y} u^{y} n^{y-z}=u_{1}^{x}
$$

or equivalently

$$
\left[\left(u^{2}+4\right)^{z}-1\right]-\left(u_{1}^{x}-1\right)=2^{2 y} u^{y} n^{y-z},
$$

with $k(z-x)=m x$, and $n^{m}=u_{2}^{k}$.

Clearly $u_{2}>1$. Assume now $u_{1}=1$. As $u^{2} \equiv 1(\bmod 8)$, by comparing $v_{2}(\cdot)$ both sides of $(20)$ and by $(1)$ of Lemma 6 we have $v_{2}\left[\left(u^{2}+4\right)^{z}-1\right]=2+v_{2}(z)<2 y$. So (21) is inconsistent. So $u_{1}>1$ and

$$
v_{2}(z)=v_{2}\left(u_{1}^{x}-1\right)-2 .
$$

If $u_{1} \equiv 1 \bmod 8$, then by (1) of Lemma 6 we get $v_{2}(z)=v_{2}\left(u_{1}-1\right)+v_{2}(x)-2$.

If $u_{1} \equiv 7 \bmod 8$ and $x$ is odd, then by (2) of Lemma $6: v_{2}\left(u_{1}^{x}-1\right)=1$, impossible by (22). Thus (21) is inconsistent.

If $u_{1} \equiv 7$ mod 8 and $x$ is even, then by (3) of Lemma $6: v_{2}\left(u_{1}^{x}-1\right)=v_{2}\left(u_{1}+1\right)+v_{2}(x)$. Hence by (22) one gets $v_{2}(z)=v_{2}\left(u_{1}+1\right)+v_{2}(x)-2$.

For $u_{1} \equiv 3 \bmod 8$, we have $v_{2}\left(u_{1}^{x}-1\right)=1$, if $x$ is odd (by (2) of Lemma 6), and $v_{2}\left(u_{1}^{x}-1\right)=2+v_{2}(x)$, if $x$ is even (by (3) of Lemma 6). Hence for (20) to be consistent one has necessarily $v_{2}(z)=v_{2}(x)$, which implies $v_{2}(z-x) \geq v_{2}(x)+1$. So from the second equation of (19): $n^{z-x}=u_{2}^{x}$ it follows that $u_{2}$ must be a square, hence $u_{2} \equiv 1 \bmod 8$. Thus $u_{1} u_{2} \equiv 3 \bmod 8$, a contradiction with $u_{1} u_{2}=u^{2}-4 \equiv 5 \bmod 8$.

Similarly, for $u_{1} \equiv 5 \bmod 8$, by using (1) of Lemma 6 we have $v_{2}\left(u_{1}^{x}-1\right)=2+v_{2}(x)$, and by the same reason $v_{2}(z)=v_{2}(x)$. Hence the system (19) is inconsistent, if $u_{2}$ is not a square.

Lemma 8. In the notations above if $x, y, z$ are even, then (20) is inconsistent.

Proof. In this case we can rewrite (20) in the form of Pythagorian equation

$$
\left(u_{1}^{x / 2}\right)^{2}+\left[2^{y} u^{y / 2} n^{(y-z) / 2}\right]^{2}=\left[\left(u^{2}+4\right)^{z / 2}\right]^{2} .
$$

Hence (cf. (1)) there are integers $X, Y$, say with $2 \mid Y$ such that

$$
\begin{aligned}
& \left(u^{2}+4\right)^{z / 2}=X^{2}+Y^{2}, \\
& 2^{y} u^{y / 2} n^{(y-z) / 2}=2 X Y .
\end{aligned}
$$

In view of Lemma 2.2 of [9], Equation (23) has solutions

$$
\begin{aligned}
& u^{2}+4=A^{2}+B^{2}, 2 \mid B, \\
& v_{2}(Y)=v(z / 2)+v_{2}(B) .
\end{aligned}
$$

Since $u^{2}+4 \equiv 5 \bmod 8$ it follows from $(25)$ that $v_{2}(B)=1$. From (24) we have $v_{2}(Y)=y-1$ which together with (26) implies

$$
y=v_{2}(z)+1,
$$

a contradiction with $y>z$.

Corollary 2. In the notations above if $y, z$ are even and $(20)$ is consistent, then $x$ is odd and $u_{1} \equiv 1(\bmod 8)$. Moreover $u_{1}$ admits a proper decomposition $u_{1}=t_{1} t_{2}$ such that $\operatorname{gcd}\left(t_{1}, t_{2}\right)=1$ and

$$
t_{2}^{x}+t_{1}^{x}=2\left(u^{2}+4\right)^{z / 2}
$$




$$
\begin{gathered}
t_{2}^{x}-t_{1}^{x}=2^{y+1} u^{y / 2} n^{(y-z) / 2}, \\
v_{2}\left(t_{1}^{x}-1\right)=v_{2}\left(t_{2}^{x}-1\right)=v_{2}\left(u_{1}^{x}-1\right)-1 .
\end{gathered}
$$

Proof. By Lemma $8 x$ is odd. In fact one can rewrite (20) as

$$
A \cdot B=u_{1}^{x} \text { with } \operatorname{gcd}(A, B)=1,
$$

where

$$
A=\left(u^{2}+4\right)^{z / 2}-2^{y} u^{y / 2} n^{(y-z) / 2}, \quad B=\left(u^{2}+4\right)^{z / 2}+2^{y} u^{y / 2} n^{(y-z) / 2} .
$$

Hence

$$
A=t_{1}^{x}, B=t_{2}^{x} \text { with } u_{1}=t_{1} t_{2} \text { and } \operatorname{gcd}\left(t_{1}, t_{2}\right)=1 .
$$

If $t_{1}=1$, then by (1) of Lemma 6: $v_{2}\left[\left(u^{2}+4\right)^{z / 2}-1\right]=2+v_{2}(z / 2)<y=v_{2}\left(2^{y} u^{y / 2} n^{(y-z) / 2}\right)$. So $A=1$ is impossible.

Now from (30) we have two possibilities:

(1) $z / 2$ is odd: $t_{1} \equiv t_{2} \equiv 5(\bmod 8)$;

(2) $z / 2$ is even: $t_{1} \equiv t_{2} \equiv 1(\bmod 8)$;

both of them imply $u_{1} \equiv 1(\bmod 8)$.

Also (27)-(29) follow immediately from (30).

Corollary 3. In the situation of Corollary 2 we have $t_{1}, t_{2} \equiv 5(\bmod 8)$ and $v_{2}\left(u_{1}-1\right)=3$.

Proof. We will show that $z / 2$ must be odd, from which the conclusion immediately follows by the proof above, noting that $v_{2}\left(u_{1}-1\right)=v_{2}\left(u_{1}^{x}-1\right)=v_{2}(A-1)+1=3$.

Assume on the contrary that $v_{2}(z) \geq 2$. In view of (30) one has $x \geq 3$, as $t_{1}<t_{2}<u^{2}-4$. We claim that $x>3$. Indeed, if $x=3$, then $n=u_{2}^{3}$ by (19), noting that $z=4$ by $B=t_{2}^{x}$ of (30), so $y=6$ as $A=t_{1}^{x}>0$. Now from the equation $t_{1}^{x}=A$ in (30) we see that $\left(t_{1}, 4 u u_{2}, u^{2}+4\right)$ is a primitive solution of

$$
X^{3}+Y^{3}=Z^{2} .
$$

Euler ([14], pp. 578-579) indicated a primitive parameterization for the Diophantine Equation (31) with $3 \nmid Z, 2 \mid Y$ as follows

$$
X=(s-t)(3 s-t)\left(3 s^{2}+t^{2}\right), \quad Y=4 s t\left(3 s^{2}-3 s t+t^{2}\right),
$$

with $s, t$ co-prime, $3 \nmid t$ and $s \not \equiv t(\bmod 2)$. Hence $8 \mid Y$ which shows that $t_{1}^{x}=A$ in $(30)$ is impossible.

Furthermore, if $x \geq 4$, then by Theorem 1.1 of [15], (27) is again impossible.

\section{Proof of Theorem 2}

The aim of this section is to show that the case $u_{1} \equiv 7(\bmod 8)$ in Lemma 7 is not realized. We refer the reader to [16] for basic properties of Jacobi quadratic and quartic residue symbols $\left(\frac{\mathrm{m}}{)},\left(\frac{\mathrm{m}}{\mathrm{m}}\right)_{4}\right.$ we shall use in the following lemmas.

Lemma 9. For a prime $p \mid\left(u^{2}+4\right)$ one has $p \equiv 1(\bmod 4)$ and $\left(\frac{u}{p}\right)=1$.

Proof. Since $u^{2} \equiv-4(\bmod p)$, so $\left(\frac{-1}{p}\right)=1$, i.e., $p \equiv 1(\bmod 4)$. Furthermore we include the following simple argument due to the referee instead of ours in the original version:

$$
\left(\frac{u}{p}\right)=\left(\frac{4 u}{p}\right)=\left(\frac{4 u+u^{2}+4}{p}\right)=\left(\frac{(u+2)^{2}}{p}\right)=1 .
$$

Lemma 10. If (20) is consistent and $u_{1} \equiv \pm 1(\bmod 8)$, then $\left(\frac{n}{p}\right)=\left(\frac{u_{2}}{p}\right)$ for any prime $p$. 
Proof. Indeed, in this case by Lemma $7 v_{2}(z)>v_{2}(x)$. Hence $v_{2}(z-x)=v_{2}(x)$, so we have in (21) $n^{m}=u_{2}^{k}$ with $k, m$ odd, and therefore the conclusion of Lemma 10.

We are ready now to prove Theorem 2. Let $p \mid\left(u^{2}+4\right)$. By taking $\left(\frac{-}{p}\right)$ on (20) and using Lemmas 9, 10 one sees that

$$
\left(\frac{u_{1}}{p}\right)^{x}=\left(\frac{n}{p}\right)^{y-z}=\left(\frac{u_{2}}{p}\right)^{y-z}=\left(\frac{u_{2}}{p}\right)^{y},
$$

(as $z$ is even). Now taking the product of (32) over all (not necessarily distinct) prime divisors $p \mid\left(u^{2}+4\right)$ we have

$$
\left(\frac{u_{1}}{u^{2}+4}\right)^{x}=\prod_{p \mid\left(u^{2}+4\right)}\left(\frac{u_{1}}{p}\right)^{x}=\prod_{p \mid\left(u^{2}+4\right)}\left(\frac{u_{2}}{p}\right)^{y}=\left(\frac{u_{2}}{u^{2}+4}\right)^{y} .
$$

By the quadratic reciprocity law

$$
\begin{aligned}
& \left(\frac{u_{1}}{u^{2}+4}\right)=\left(\frac{u^{2}+4}{u_{1}}\right)=\left(\frac{2}{u_{1}}\right)=1 \\
& \left(\frac{u_{2}}{u^{2}+4}\right)=\left(\frac{u^{2}+4}{u_{2}}\right)=\left(\frac{2}{u_{2}}\right)=-1
\end{aligned}
$$

as $u_{1} \equiv \pm 1(\bmod 8), u_{2} \equiv \pm 5(\bmod 8)$. Altogether (33)-(35) imply that $\left(\frac{u_{2}}{p}\right)^{y}=(-1)^{y}=1$, i.e., $y$ must be even.

Corollary 4. The possibility $u_{1} \equiv 7(\bmod 8)$ in Lemma 7 is not realized.

Proof. Indeed, in this case $v_{2}(z)>v_{2}(x) \geq 1$, so (20) is inconsistent by Lemma 8 .

Corollary 5. In the case $u_{1} \equiv 1(\bmod 8)$ of Lemma 7 we have

$$
\left(v_{2}(x), v_{2}(y), v_{2}(z)\right)=(0, \geq 2,1) .
$$

Proof. By Lemma 7 and Theorem 2: $y, z$ are even, hence $x$ is odd by Lemma 8. From the proof of Corollary 3 it follows that $v_{2}(z)=1$. For a prime $p \mid\left(u^{2}+4\right)$ by taking $\left(-\frac{1}{p}\right)$ on $A=t_{1}^{x}$ of (30) and using Lemma 9 one gets

$$
\left(\frac{t_{1}}{p}\right)=\left(\frac{n}{p}\right)^{(y-z) / 2}
$$

By the same reason of (35) we have $\left(\frac{t_{1}}{u^{2}+4}\right)=-1$, as $t_{1} \equiv 5(\bmod 8)$ by Corollary 3. Hence there exists a prime $p_{0} \mid\left(u^{2}+4\right)$ such that

$$
\left(\frac{t_{1}}{p_{0}}\right)=-1
$$
5 follows.

From (36), (37) one concludes that $(y-z) / 2$ must be odd (and $\left(\frac{n}{p_{0}}\right)=-1$ ), so the conclusion of Corollary

Remark 2. One can have another proof of Lemma 8 as shown in several steps below. Assuming $y, z$ even, and arguing as in the proof of Corollary 2 one gets Equation (30) together with (27)-(29).

1) If $u_{1} \equiv 5(\bmod 8)$ we have four possibilities for $\left(t_{1}, t_{2}\right)$ :

(i) $t_{1} \equiv 1(\bmod 8), t_{2} \equiv 5(\bmod 8)$;

(ii) $t_{1} \equiv 5(\bmod 8), t_{2} \equiv 1(\bmod 8)$;

(iii) $t_{1} \equiv 3(\bmod 8), t_{2} \equiv 7(\bmod 8)$;

(iv) $t_{1} \equiv 7(\bmod 8), t_{2} \equiv 3(\bmod 8)$;

all of them violate (29). 
(2) Assume now $u_{1} \equiv \pm 1(\bmod 8)$ and $x$ even, hence $v_{2}(z) \geq 2$ by Lemma 7 . We will shows that $v_{2}(y)=1$. Indeed, considering $p \mid\left(u^{2}+4\right)$ and taking $\left(\frac{-}{p}\right)_{4}$ on (20) one has by using Lemmas 9, 10

$$
\left(\frac{u_{1}}{p}\right)^{x / 2}=\left(\frac{-1}{p}\right)_{4}\left(\frac{n}{p}\right)^{(y-z) / 2}=\left\{\begin{array}{c}
\left(\frac{u_{2}}{p}\right)^{y / 2}, p \equiv 1(\bmod 8) \\
-\left(\frac{u_{2}}{p}\right)^{y / 2}, p \equiv 5(\bmod 8)
\end{array}\right.
$$

as $z / 2$ is even. Let $r$ denote the number of prime divisors $p \mid\left(u^{2}+4\right), p \equiv 5(\bmod 8)$. Clearly $r$ is odd, as $u^{2}+4 \equiv 5(\bmod 8)$. In a similar way as in (33)-(35), taking the product of (38) over all (not necessarily distinct) prime divisors $p \mid\left(u^{2}+4\right)$ we get

$$
1=\left(\frac{u_{1}}{u^{2}+4}\right)^{x / 2}=(-1)^{r}\left(\frac{u_{2}}{u^{2}+4}\right)^{y / 2}=-(-1)^{y / 2}
$$

Hence $y / 2$ must be odd, so $(y-z) / 2$ is odd. For any prime $p \mid\left(u^{2}+4\right)$ taking $\left(\frac{-}{p}\right)$ on equation $A=t_{1}^{x}$ from (30) now gives us

$$
\left(\frac{n}{p}\right)=1\left(=\left(\frac{u_{2}}{p}\right) \text { by Lemma } 10\right)
$$

On the other hand from (35) it follows that there exists a prime $p_{0} \mid\left(u^{2}+4\right)$ such that $\left(\frac{u_{2}}{p_{0}}\right)=-1$, a contradiction with (39). Thus (30) (and hence (20)) is inconsistent.

\section{The case $u_{1} \equiv 5(\bmod 8)$}

In this case by (3) of Lemma 7 we have $v_{2}(z)=v_{2}(x)$, hence from (19) it follows that $u_{2}=w^{2^{s}}$, where $s=v_{2}(z-x)-v_{2}(x)$. The following lemma can be proved similarly as Lemma 10.

Lemma 11. If $(20)$ is consistent and $u_{1} \equiv 5(\bmod 8)$, then $\left(\frac{n}{p}\right)=\left(\frac{w}{p}\right)$ for any prime $p$.

Proof. Indeed, in this case $n^{m}=w^{k}$ with $k, m$ odd by the above argument, and therefore the conclusion of Lemma 11.

Lemma 12. If $x, z$ are even and $(20)$ is consistent, then $y$ is odd and $u_{1} \equiv 5(\bmod 8)$. Moreover $n$ admits a decomposition $n=n_{1} n_{2}$ such that $\operatorname{gcd}\left(n_{1}, n_{2}\right)=1$ and

$$
\left\{\begin{array}{l}
u_{1}^{x / 2}=u^{y} n_{2}^{y-z}-2^{2 y-2} n_{1}^{y-z} \\
\left(u^{2}+4\right)^{z / 2}=u^{y} n_{2}^{y-z}+2^{2 y-2} n_{1}^{y-z}
\end{array}\right.
$$

Proof. By Lemma $8 y$ is odd. In view of Lemma 7 and Theorem 2 we are in the situation (3) of Lemma 7. Now one rewrites (20) as

$$
C_{1} \cdot D_{1}=2^{2 y} u^{y} n^{y-z} \text { with } \operatorname{gcd}\left(C_{1}, D_{1}\right)=2,2 \| D_{1}
$$

where

$$
C_{1}=\left(u^{2}+4\right)^{z / 2}-u_{1}^{x / 2}, D_{1}=\left(u^{2}+4\right)^{z / 2}+u_{1}^{x / 2} .
$$

As $2 \| D_{1}$ we obtain either

$$
C_{1}=2^{2 y-1} n_{1}^{y-z}, \quad D_{1}=2 u^{y} n_{2}^{y-z},
$$

or

$$
C_{1}=2^{2 y-1} u^{y} n_{1}^{y-z}, \quad D_{1}=2 n_{2}^{y-z},
$$

where $n=n_{1} n_{2}, \operatorname{gcd}\left(n_{1}, n_{2}\right)=1$ and

$$
w=w_{1} w_{2}, n_{1}^{m}=w_{1}^{k}, n_{2}^{m}=w_{2}^{k}
$$


with $k, m$ odd from Lemma 11. Note that this is not used in the proof here, we label it for convenience in proving Proposition 1 below.

Clearly (41) is equivalent to (40). It remains to show that (42) can't happen by rewriting it as

$$
\left\{\begin{array}{l}
u_{1}^{x / 2}=n_{2}^{y-z}-2^{2 y-2} u^{y} n_{1}^{y-z}, \\
\left(u^{2}+4\right)^{z / 2}=n_{2}^{y-z}+2^{2 y-2} u^{y} n_{1}^{y-z},
\end{array}\right.
$$

which is impossible, since $\left(u^{2}+4\right)^{z / 2}<2^{2 y-2} u^{y}$.

Lemma 13. If $\left(\frac{u_{1}}{u}\right)=1$ and $u_{2}$ is a square, then $u \equiv 1(\bmod 4)$.

Proof. We have obviously

$$
1=\left(\frac{u_{1}}{u}\right)=\left(\frac{u_{1} u_{2}}{u}\right)=\left(\frac{u^{2}-4}{u}\right)=\left(\frac{-1}{u}\right),
$$

so the conclusion of the lemma.

Lemma 14. In the notations of Lemma 11 we have

(1) if $w \equiv \pm 3(\bmod 8)$, then $x, z$ are odd, $y$ is even;

(2) if $w \equiv \pm 1(\bmod 8)$, then $x, z$ are even, $y$ is odd.

Proof. For a prime $p \mid\left(u^{2}+4\right)$ by taking $\left(\frac{-}{p}\right)$ on (20) and using Lemmas 9, 11 one sees that

$$
\left(\frac{u_{1}}{p}\right)^{x}=\left(\frac{n}{p}\right)^{y-z}=\left(\frac{w}{p}\right)^{y-z} .
$$

By taking the product of both sides of (45) over all (not necessarily distinct) prime divisors $p \mid\left(u^{2}+4\right)$ and using the reciprocity law we have

$$
\begin{gathered}
\prod_{p \mid\left(u^{2}+4\right)}\left(\frac{u_{1}}{p}\right)^{x}=\left(\frac{u_{1}}{u^{2}+4}\right)^{x}=\left(\frac{u^{2}+4}{u_{1}}\right)^{x}=\left(\frac{2}{u_{1}}\right)^{x}=(-1)^{x}, \\
\prod_{p \mid\left(u^{2}+4\right)}\left(\frac{w}{p}\right)^{y-z}=\left(\frac{w}{u^{2}+4}\right)^{y-z}=\left(\frac{u^{2}+4}{w}\right)^{y-z}=\left(\frac{2}{w}\right)^{y-z}= \begin{cases}(-1)^{y-z}, & w \equiv \pm 3(\bmod 8), \\
1, & w \equiv \pm 1(\bmod 8) .\end{cases}
\end{gathered}
$$

Hence if $w \equiv \pm 3(\bmod 8)$, then by equalizing (46), (47): $(-1)^{x}=(-1)^{y-z}$. Thus $y$ must be even, as $v_{2}(z)=v_{2}(x)$. In view of Lemma $8 x, z$ are odd.

In the case $w \equiv \pm 1(\bmod 8)$, again equalizing (46), (47) we see that $(-1)^{x}=1$, therefore $x$ is even, and so is $z$. By Lemma $8 y$ must be odd.

Proposition 1. In the situation of Lemma 14 we have

(1) if $w \equiv \pm 3(\bmod 8)$, then $u \equiv 1(\bmod 4)$;

(2) if $w \equiv \pm 1(\bmod 8)$, then $u \equiv \pm 3(\bmod 8)$. Moreover, if $u \equiv 3(\bmod 8)$, then $w$ can not be a square.

Proof. (1) If $w \equiv \pm 3(\bmod 8)$, then $x, z$ are odd in view of Lemma 14. So by taking $\left(\frac{-}{u}\right)$ on (20) one gets $\left(\frac{u_{1}}{u}\right)=1$, hence $u \equiv 1(\bmod 4)$ by Lemma 13 .

(2) In the case $w \equiv \pm 1(\bmod 8): x, z$ are even, $y$ is odd by Lemma 14 . There are two subcases to consider.

I. $x / 2, z / 2$ are odd. For a prime $p \mid\left(u^{2}+4\right)$ by taking $\left(\frac{-}{p}\right)$ on $D_{1}=2 u^{y} n_{2}^{y-z}$ from (41), (43) and using Lemmas 9,11 one sees that

$$
\left(\frac{u_{1}}{p}\right)=\left(\frac{2}{p}\right)\left(\frac{n_{2}}{p}\right)=\left(\frac{2}{p}\right)\left(\frac{w_{2}}{p}\right)= \begin{cases}\left(\frac{w_{2}}{p}\right), & p \equiv 1(\bmod 8), \\ -\left(\frac{w_{2}}{p}\right), & p \equiv 5(\bmod 8) .\end{cases}
$$


Recall that the number of (not necessarily distinct) prime divisors $p \mid\left(u^{2}+4\right), p \equiv 5(\bmod 8)$ is odd, so $\prod_{p \mid\left(u^{2}+4\right)}\left(\frac{2}{p}\right)=-1$. Now taking the product of both sides of (48) over all (not necessarily distinct) prime divisors $p \mid\left(u^{2}+4\right)$ and using the reciprocity law one has

$$
\prod_{p \mid\left(u^{2}+4\right)}\left(\frac{u_{1}}{p}\right)=\left(\frac{u_{1}}{u^{2}+4}\right)=\left(\frac{u^{2}+4}{u_{1}}\right)=\left(\frac{2}{u_{1}}\right)=-1,
$$

and

$$
\prod_{p \mid\left(u^{2}+4\right)}\left(\frac{2}{p}\right)\left(\frac{w_{2}}{p}\right)=-\prod_{p \mid\left(u^{2}+4\right)}\left(\frac{w_{2}}{p}\right)=-\left(\frac{w_{2}}{u^{2}+4}\right)=-\left(\frac{u^{2}+4}{w_{2}}\right)=-\left(\frac{2}{w_{2}}\right) .
$$

Equalizing (49), (50) we get $w_{2} \equiv \pm 1(\bmod 8)$, so in view of $(43): n_{2} \equiv \pm 1(\bmod 8)$. From this and (40) it follows that $u \equiv \pm 3(\bmod 8)$. Moreover, if $u \equiv 3(\bmod 8)$, then $w_{2} \equiv-1(\bmod 8)$, hence by

II. $x / 2, z / 2$ are even. If one takes $\left(\frac{-}{u}\right)$ on the second equation of $(40)$, then $\left(\frac{n_{1}}{u}\right)=1$. Now taking
. $\left(\frac{-}{u}\right)$ on the first equation of $(40)$ we get $1=\left(\frac{-1}{u}\right)\left(\frac{n_{1}}{u}\right)$. Thus $u \equiv 1(\bmod 4)$.

The proof of Proposition 1 is completed.

As for Theorem 3 notice that the case $u_{1} \equiv \pm 1(\bmod 8)$ follows from Corollaries $2,3,4$ and 5 . The rest of Theorem 3, i.e., the case $u_{1} \equiv 5(\bmod 8)$, follows from Lemma 14 and Proposition 1.

The equalities for Jacobi symbols are immediate from (20) and Lemma 11.

\section{Proof of Corollary 1}

In this section we shall apply results of previous parts for establishing the truth of Jeśmanowicz' conjecture for $u<100$ and $v=2$. In view of Theorem 3 one has to consider only two cases: $u_{1} \equiv 1(\bmod 8)$ and $u_{1} \equiv 5(\bmod 8)$.

Observation 1. If $u_{1} \equiv 1(\bmod 8)$ and $(20)$ is consistent, then $u>183$.

Proof. Indeed, it was noted that $x \geq 3$ by (30). On the other hand from the proof of Corollary 3 we have $v_{2}(z)=1$, so $z \geq 6$, hence $y \geq 8$. From (28) it follows that $2^{y+1} \mid t_{2}-t_{1}$, as $x$ is odd. Since $t_{1}, u_{2}$ are co-prime and $\equiv 5(\bmod 8)$, so $t_{1} u_{2} \geq 5 \cdot 13$. Therefore $u>\sqrt{t_{1} t_{2} u_{2}} \geq \sqrt{\left(2^{9}+5\right) \cdot 65}>183$.

Observation 2. If $u_{1} \equiv 1(\bmod 8)$ and $(20)$ is consistent, then in fact $u>729$.

Proof. By Corollary 5 one knows $4 \mid y$. We claim that $y \geq 12$. Assuming on the contrary $y=8$, then by the above $z=6$. In view of (27) and [17] we must have $x>3$, so $x=5$, which gives us a non-trivial solution of $X^{5}+Y^{5}=2 Z^{3}$. This is impossible by [18] (Theorem 1.5).

Therefore $y \geq 12$, and by the argument above $u>\sqrt{\left(2^{13}+5\right) \cdot 65}>729$.

It remains to consider the case $u_{1} \equiv 5(\bmod 8)$. In the range of odd primes $<100$ there are ten possibilities with $u^{2}-4=u_{1} u_{2}$ and $u_{2}$ is a square, namely $u=7,11,23,43,47,61,73,79,83,97$. In view of Proposition 1 we shall exclude the possibilities $u=7,23,47,79$.

Observation 3. For $\left(u, u_{1}, u_{2}\right)=\left(11,13,3^{2}\right),\left(43,5 \cdot 41,3^{2}\right),\left(83,5 \cdot 17,3^{4}\right)$ we have $w \equiv \pm 3(\bmod 8)$, hence $u \equiv$ $1(\bmod 4)$ by Proposition 1 , a contradiction. Note that in the original version to eliminate the possibility $\left(83,5 \cdot 17,3^{4}\right)$ and $w=9$ we used implicitly the fact that if $u \equiv 3(\bmod 8)$, then $w$ can not be a square, which we include a proof in the revised version (cf. Proposition 1 above). The referee provides another argument by choosing $p=5 \mid u_{1}$ which leads also to a contradiction as follows

$$
1=\left(\frac{9}{5}\right)=\left(\frac{w}{p}\right) \neq\left(\frac{u}{p}\right)=\left(\frac{83}{5}\right)=-1 .
$$

Observation 4. For $\left(u, u_{1}, u_{2}\right)=\left(61,7 \cdot 59,3^{2}\right)$ one has $w=3$, so

$$
-1=\left(\frac{w}{7}\right) \neq\left(\frac{u^{2}+4}{7}\right)=1,
$$


a contradiction with (2.1) of Theorem 3.

Observation 5. For $\left(u, u_{1}, u_{2}\right)=\left(73,3 \cdot 71,5^{2}\right)$ we have $w=5$, hence $x, z$ are odd and $y$ is even by (2.1) of Theorem 3. Taking modulo 73 on (20) one gets

$$
4^{z} \equiv(-6)^{x}(\bmod 73)
$$

Working in $\mathbb{F}_{73}^{*}$ we have

$$
\operatorname{ord}(4)=9, \quad \operatorname{ord}(-6)=36 .
$$

Therefore from (51), (52) it follows that $36 \mid 9 x$, so $4 \mid x$, a contradiction.

Observation 6. For $\left(u, u_{1}, u_{2}\right)=\left(97,5 \cdot 11 \cdot 19,3^{2}\right)$ one has $w=3$, so

$$
1=\left(\frac{w}{11}\right) \neq\left(\frac{u^{2}+4}{11}\right)=-1,
$$

again a contradiction with (2.1) of Theorem 3.

Acknowledgments: The authors would like to thank the referee for many valuable comments and suggestions greatly improving the content of the paper.

Author Contributions: All authors contributed equally to the writing of this paper. All authors read and approved the final manuscript.

Conflicts of Interest: "The authors declare no conflict of interest".

\section{References}

[1] Jeśmanowicz, L. (1955). Several remarks on Pythagorean numbers. Wiadom. Mat, 1(2), 196-202, (in Polish).

[2] Le, M.-H., Scott, R., \& Styer, R. (2019). A survey on the ternary purely exponential Diophantine equation $a^{x}+b^{y}=c^{z}$. Surveys in Mathematics \& its Applications, 14, 109-140.

[3] Deng, M.-J., \& Cohen, G. L. (1998). On the conjecture of Jeśmanowicz concerning Pythagorean triples. Bulletin of the Australian Mathematical Society, 57(4), 515-524

[4] Le, M.-H. (1999). A note on Jeśmanowicz' conjecture concerning Pythagorean triples. Bulletin of the Australian Mathematical Society, 59(3), 477-480.

[5] Yang, H., \& Fu, R. (2015). A note on Jeśmanowicz' conjecture concerning primitive Pythagorean triples. Journal of Number Theory, 156(1), 183-194.

[6] Zsigmondy, K. (1892). Zur theorie der potenzreste. Monatshefte für Mathematik und Physik, 3(1), 265-284.

[7] Birkhoff, G. D., \& Vandiver, H. S. (1904). On the integral divisors of $a^{n}-b^{n}$. Annals of Mathematics, 5(4), 173-180.

[8] Carmichael, R. D. (1913). On the numerical factors of the arithmetic forms $\alpha^{n} \pm \beta^{n}$. The Annals of Mathematics, 15(2), $30-70$.

[9] X.-W. Zhang, W.-P. Zhang, The exponential diophantine equation $\left(\left(2^{2 m}-1\right) n\right)^{x}+\left(2^{m+1} n\right)^{y}=\left(\left(2^{2 m}+1\right) n\right)^{z}$, Bull. Math. Soc. Math. Roum., Nouv. Sér., 57:3 (2014), 337-344.

[10] Miyazaki, T. (2015). A remark on Jeśmanowicz' conjecture for the non-coprimality case. Acta Mathematica Sinica, English Series, 31(8), 1255-1260.

[11] Terai, N. (2014). On Jeśmanowicz' conjecture concerning primitive Pythagorean triples. Journal of Number Theory, 141(2), 316-323.

[12] Deng, M.-J. (2014). A note on the diophantine equation $(n a)^{x}+(n b)^{y}=(n c)^{z}$. Bulletin of the Australian Mathematical Society, 89(2), 316-321.

[13] Yang, H., Ren, R.-Z., \& Fu, R.-Q. (2017). On Jeśmanowicz' conjecture concerning Pythagorean numbers. Math. J. Wuhan, 37(3), 506-512, (in Chinese).

[14] Dickson, L. E. (1971). History of the Theory of Numbers, Vol. 2. Chelsea Publishing Company, New York.

[15] Bennett, M. A., \& Skinner, C. M. (2004). Ternary Diophantine equations via Galois representations and modular forms. Canadian Journal of Mathematics, 56(1), 23-54.

[16] Ireland, k., \& Rosen, M. (1982). A Classical Introduction to Modern Number Theory. Springer.

[17] Wakulicz, A. (1958). On the equation $x^{3}+y^{3}=2 z^{3}$. Colloquium Mathematicum, 5, 11-15.

[18] Bennett, M. A., Vatsal, V., \& Yazdani, S. (2004). Ternary Diophantine equations of signature $(p, p, 3)$. Compositio Mathematica, 140(6), 1399-1416. 
(C) 2021 by the authors; licensee PSRP, Lahore, Pakistan. This article is an open access article distributed under the terms and conditions of the Creative Commons Attribution (CC-BY) license (http://creativecommons.org/licenses/by/4.0/). 